\title{
An Analysis of the Influence of Need for Cognition on Dynamic Queries Usage
}

\author{
Giuseppe Carenini \\ Department of Computer Science \\ University of British Columbia \\ 2366 Main Mall \\ Vancouver, B.C. Canada V6T $1 Z 4$ \\ $+1604822-510$ \\ carenini@cs.ubc.ca
}

\begin{abstract}
We report preliminary results about a positive correlation between the personality trait of need for cognition (a construct developed in social psychology) and the usage of dynamic queries (an interactive technique for database querying).
\end{abstract}

\section{Keywords}

Interactive techniques, user differences, empirical testing.

\section{INTRODUCTION}

A growing body of research in HCI is devoted to investigate visualization and interactive techniques [4], which can substantially improve the performance of systems for data exploration and analysis. These techniques enable users to perceive relationships and manipulate datasets, replacing more demanding cognitive operations with fewer and more efficient perceptual and motor operations. Typically, when researches devise a new technique they evaluate its effectiveness by means of a taskbased comparison. The performance of subjects applying the new technique on a pool of data exploration tasks is compared with the performance of subjects applying traditional techniques on the same tasks (e.g., [1]). Although this kind of comparative evaluation is crucial to stimulate the adoption of novel techniques, we believe that additional user studies are needed. In particular, given that new, general-purpose interactive techniques will be used in an ever-increasing number of applications by an everincreasing number of user communities, it becomes critical to understand whether (and to what extent) the usage of these techniques is influenced by differences between and within user populations. These differences may include, for instance, cognitive and personality traits such as users' intelligence, curiosity, visual memory, memory span and perceptual speed.

In this paper, we give preliminary results about the influence of a personality trait known as Need For
Cognition (NFC) [2] on the usage of Dynamic Queries (DQs) [5]. NFC is a construct, developed in social psychology, which measures the subject's tendency to engage and enjoy effortful cognitive tasks. NFC has been proven to be a stable individual trait largely independent of psychometric intelligence and showing significant positive correlation with school performance, curiosity and thoughtful examination of arguments.

DQs are a highly successful interactive technique for database querying. Several studies have shown that DQs produce dramatic performance improvements and high levels of user satisfaction. Thus, we expect them to become a key component of an increasing number of interfaces for data exploration and analysis serving a large spectrum of diverse user communities. The need to understand how DQs usage is influenced by users' traits is therefore particularly pressing.

\section{METHODS}

Our preliminary analysis is based on log files collected while 38 users interacted with the interface for exploring real-estate information shown in Figure 1. The users' NFC was collected through a standard questionnaire [3], before they interacted with the interface. As part of a larger experiment, our subjects were given the task to select out of a set of available houses the four they liked most given the information shown in the interface. Notice that the map shows the house's location (houses are represented as marks), the bar charts show the size of amenities and the table specifies house's attributes related to its quality. To facilitate users in the exploration of the dataset, the realestate interface provides the user with a repertoire of interactive techniques. Because of the limited scope of this paper, we only describe DQs. The four rectangular objects above the map are DQs for the attributes "distance from University", "distance from rapid transportation", "distance from park", and "distance from shopping". They allow the user to restrict, in a rapid, incremental and reversible way, the set of entities shown on the visualization frames to those entities whose value for an attribute is in a given range. The user can specify any range of values for an attribute by 


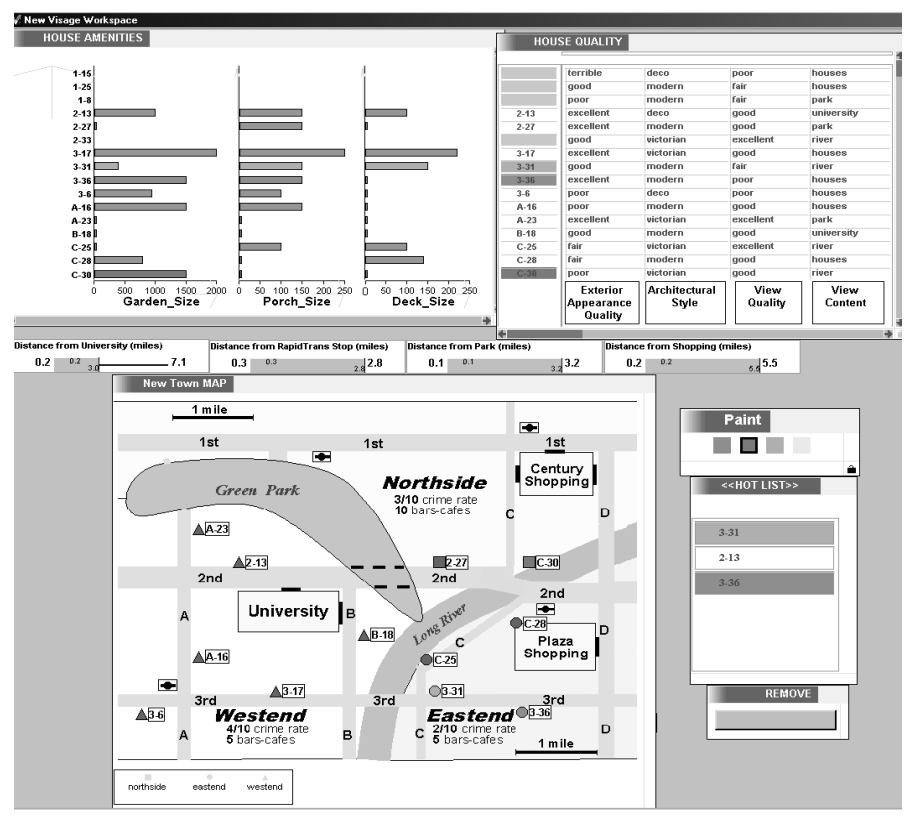

Figure 1 The interface for data exploration

sliding a drag box or its edges with the mouse. For example, in Figure 1, the user expresses preference for houses not further than 3 miles from the University. The result of this selection is that all houses not satisfying the constraint are grayed in the table and disappeared both from the map and from the bar charts.

\section{RESULTS}

The results of our analysis are summarized in the table. Time on task corresponds to the time a user spent on the exploration task previously described, Number of actions (no $D Q$ ) measures how many explorative actions the user performed excluding the use of DQs, and finally $D Q$ usage measures how many times the user slid the slider of a DQ. Each cell in the table indicates the Pearson $r$ correlation between the users' NFC and the column variable, for the user population reported in parenthesis. In the first row, we consider all the 38 users. As shown in the table, we found a modest correlation between NFC and DQ usage. Since this does not appear to be due to either more time spent on the task, or to a general tendency to perform more actions (consider the much lower correlations with these two variables), we performed further analysis. In the second row, we focus only on the 32 users who actually did use the DQs at least once. As shown, the correlation between NFC and DQ usage becomes much stronger and marginally significant. Finally, as can be seen in the third row, if we remove the user with highest NFC from the analysis ( $\mathrm{s} / \mathrm{he}$ appears to be an outlier) the correlation becomes even stronger and statistically significant.

\section{DISCUSSION}

Our analysis seems to indicate that the users' NFC influences how intensely they will use DQs. This finding has several implications for the development of interfaces based on DQs. For instance, when performing experiments

\begin{tabular}{|c|c|c|c|}
\hline Pearson r & $\begin{array}{c}\text { Time } \\
\text { on task }\end{array}$ & $\begin{array}{c}\text { Number of } \\
\text { actions (no DQ) }\end{array}$ & DQ usage \\
\hline NFC (All Users) & 0.01 & 0.09 & 0.18 \\
\hline $\begin{array}{c}\text { NFC } \\
\text { (DQ Users) }\end{array}$ & 0.03 & 0.04 & $\begin{array}{c}\mathbf{0 . 2 9} \\
(\mathrm{p} \leq 0.10)\end{array}$ \\
\hline $\begin{array}{c}\text { NFC } \\
\text { (DQ Users - } \\
\text { outlier) }\end{array}$ & 0.09 & 0.09 & $\begin{array}{c}\mathbf{0 . 3 5} \\
(\mathrm{p} \leq 0.05)\end{array}$ \\
\hline
\end{tabular}

to evaluate an interface in which DQs (or similar techniques) play a prominent role, it is advisable to collect the subjects' NFC prior to running the experiment. A measure of the subjects NFC would allow the experimenter either to create more balanced experimental groups, or at least to perform some regression analysis at the end of the experiment. With respect to deploying an interface based on DQs, an implication of our study is that particular attention should be paid to provide training and constructive help to users low in NFC (or in any other available traits known to correlate with NFC). This special support should compensate for their tendency of not spontaneously using DQs. Finally, the correlation we have found between NFC and DQ usage could be exploited in an adaptive interface. The subject usage of DQs could represent a piece of evidence for inferring her NFC, and this assessment could be then used to adapt aspects of the interface to the subject.

\section{CONCLUSIONS}

The results described in this paper are preliminary. More empirical testing is necessary. First, an experiment involving more subjects could verify the robustness of the correlation between NFC and DQs usage. Second, further experiments should investigate whether a positive correlation also holds between NFC and the usage of other interactive techniques. Finally, as we argued at the beginning, similar experiments should be performed to test whether and how other users' cognitive and personality traits influence the usage of interactive techniques.

\section{REFERENCES}

1. Ahlberg, C., C. Williamson, and B. Shneiderman. Dynamic queries for information exploration: An implementation and evaluation. in ACM CHI'92. 1992.

2. Cacioppo, J.T. and R.E. Petty, The Need for Cognition. Journal of Personality and Social Psychology, 1982. 42: p. 116-131.

3. Cacioppo, J.T., R.E. Petty, and C.F. Kao, The efficient Assessment of need for Cognition. Journal of Personality Assessment, 1984. 48(3): p. 306-307.

4. Card, S.K., J.D. Mackinlay, and B. Shneiderman, Readings in Information Visualization - Using Vision to Think. 1999: Morgan Kaufmann.

5. Shneiderman, B., Dynamic Queries for Visual Information Seeking. IEEE Software, 1994. 11(6): p. 70-77. 